\title{
High expression of TMEM40 is associated with the malignant behavior and tumorigenesis in bladder cancer
}

\author{
Zhen-Fei Zhang 1,2, Han-Rong Zhang 1,2, Qing-Yan Zhang ${ }^{1,2,3}$, Shu-Yu Lai ${ }^{1,2}$, Yu-Zhen Feng ${ }^{1,2}$, Yi Zhou ${ }^{4}$, \\ Si-Rong Zheng ${ }^{1,2}$, Rong Shi ${ }^{1,2^{*}}$ and Jue-Yu Zhou ${ }^{1,2^{*}}$
}

\begin{abstract}
Background: Bladder cancer (BCa) is one of the most common cancers in the urinary system among the world. Previous studies suggested that TMEM40 expression level was significantly associated with clinicopathological parameters including histological grade, clinical stage and PT status of bladder cancer. However, the molecular mechanism of TMEM40 in BCa remains poorly understood.

Methods: Real-time quantitative RT-PCR (qRT-PCR) and western blot (WB) were used to examine the expression levels of TMEM40 in BCa tissues, paired non-cancer tissues and cell lines. A series of experiments, including CCK-8, wound healing, flow cytometry, transwell and EdU assays were performed to assess the effects of TMEM 40 on cell proliferation, cell cycle and apoptosis, migration and invasion. In addition, tumor growth was evaluated in vivo using a xenogenous subcutaneously implant model. All statistical analyses were executed by using the SPSS 20.0 software. All experimental data from three independent experiments were analyzed by Student's $t$ test and results were expressed as mean \pm standard deviation.

Results: In this study, we identified the role of TMEM40 in the tumorigenesis of bladder cancer and found that it was upregulated in bladder cancer tissues and cell lines, compared with their normal counterparts. The results demonstrated that effective silence of TMEM40 expression suppressed cell proliferation, blocked G1-to-S cell cycle transition, and inhibited cell migration and invasion in human bladder 5637 and EJ cell lines. Consistently, in vivo data showed that TMEM40 silencing could dramatically decreased tumor growth. Further study revealed that TMEM40 knockdown resulted in accumulation of p53 and p21 protein and decrease of c-MYC and cyclin D1 protein.
\end{abstract}

Conclusion: These data suggest that TMEM40 represents a potential oncogene, which exert a crucial role in the proliferation and apoptosis via the p53 signaling pathway in BCa, thus probably serve as a novel candidate biomarker and a potential therapeutic target for patients with BCa.

Keywords: TMEM40, Bladder cancer, Malignant phenotype, Tumorigenesis, p53 pathway

\section{Background}

Bladder cancer (BCa) is one of the most common urological malignancies across the world, especially in elderly men [1]. It is reported that there are 386,000 new cases and almost 150,000 deaths worldwide annually. $\mathrm{BCa}$ is

\footnotetext{
*Correspondence: shirongphd@126.com; zhoujueyu@126.com ${ }^{1}$ Department of Biochemistry and Molecular Biology, School of Basic Medical Sciences, Southern Medical University, Guangzhou 510515, People's Republic of China

Full list of author information is available at the end of the article
}

classified as non-muscle invasive and muscle-invasive cancers [2]. Approximately, 70\% of patients display indications for non-muscle-invasive $\mathrm{BCa}$ (NMIBC) during the initial diagnosis with mutable danger of recurrence and development to invasive diseases, hence needing long-term surveillance [3]. The recommended maintenance schedules from transurethral resection to radiation therapy and systemic chemotherapy at present are effective only in a subset of patients, and the 5-year overall survival rate remains at a low level [4]. 
Although numerous genes associated with $\mathrm{BCa}$ tumorigenesis and tumor metastasis were uncovered, the underlying molecular mechanism has not been thoroughly elucidated. Lacking of sophisticated understanding of the pathogenetic mechanism is one of the most crucial reasons for dismal outcomes in cancer patients [5]. Therefore, it is essential to explore new detailed mechanisms and molecular pathways activated in $\mathrm{BCa}$ for developing novel treatment options for anticancer therapy in $\mathrm{BCa}$.

Transmembrane protein 40 (TMEM40) is a multipass membrane protein consisting of 233 amino acids and exists two isoforms [6-9]. It is localized at chromosome 3p25.2 and is believed to play a role in collagen induced arthritis (CIA) $[10,11]$. A study reported that expression of TMEM40 were significantly higher in patients carrying Granulin (GRN) mutations compared with asymptomatic carriers and other frontotemporal lobar degeneration (FTLD) [12]. Importantly, our previous finding suggested that the expression of TMEM40 in BCa was significantly related to its pathologic grade, clinical stage, histological grade and pT status [13]. However, the specific function of TMEM40 in bladder carcinogenesis, especially its molecular mechanisms by which TMEM40 exhibits its functions and modulates the malignant behaviors of $\mathrm{BCa}$ cells, has not been fully understood.

In this study, we initially explored TMEM40 mRNA and protein expression and the correlation with malignant behavior, confirmed its potential role in proliferation, migration, and invasion of bladder cancer cells in vitro and tumorigenicity in vivo. Furthermore, we investigated the biologic functions of TMEM40 and underlying molecular mechanisms of $\mathrm{BCa}$ occurrence and progression in $\mathrm{BCa}$ cells.

\section{Methods}

\section{Patients and clinical samples collection}

$\mathrm{BCa}$ tissues and adjacent normal tissues were obtained from 12 patients, who underwent bladder surgical resection without preoperative systemic therapy in Nanfang Hospital of Southern Medical University between October 2014 and September 2016. After surgical removal, the tissues were immediately frozen using liquid nitrogen. All the patients signed informed consent and the study was approved by the Ethics Committee of Nanfang Hospital of Southern Medical University.
Cell lines and cell culture

BCa EJ, J82, BIU-87, UMUC3, SW780, 5637, T24 and normal urothelial cell line SV-HUC-1 cells used in this study were obtained from laboratory preservation. The 5637, EJ, SW780, J82 and BIU87 cells were cultured in RPMI-1640 Medium (Invitrogen, Carlsbad, CA, USA), the UMUC3, T24 cells were cultured in Dulbecco's Modified Eagle Medium (Invitrogen, Carlsbad, CA, USA), SVHUC-1 cells were cultured in F12K (Invitrogen, Carlsbad, CA, USA). All cells were plus $10 \%$ fetal bovine serum (FBS) and 1\% ampicillin (100 units $/ \mathrm{mL}$ ) and streptomycin (100 units $/ \mathrm{mL}$ ) and then placed at $37{ }^{\circ} \mathrm{C}$ with a humidified atmosphere of $95 \%$ air and $5 \% \mathrm{CO}_{2}$ in incubator.

\section{siRNA or shRNA and plasmid DNA transfection}

The specific small interfering RNA of TMEM40 and a scrambled negative control were purchased from RiboBio (Guangzhou, China). The overexpression vector pEZ-M98 and a scrambled negative control empty vector were also purchased from GeneCopoeia (Guangzhou, China). The shRNA vector and a scrambled negative control vector were obtained from Genechem (Shanghai, China). The sequence of siRNA was $5^{\prime}$-GGA UGAGCUUCAACUCUAUTT-3'; NC was $5^{\prime}$-UUCUCCG AACGUGUCACGUTT-3'; shRNA was $5^{\prime}$-GUGGACG CCUCUCAGUUAA-3'; NC was 5'-TTCTCCGAACGTG TCACGT-3';

After cells were cultured $24 \mathrm{~h}$, the cells were transiently transfected with corresponding siRNA or plasmid DNA using Lipofectamine 2000 reagents (Invitrogen, Carlsbad, CA, USA) according to the manufacturer's instructions. After $48 \mathrm{~h}$, cells transfected with siRNA or shRNA and plasmid were harvested for qRT-PCR or western blot detection.

\section{Real-time reverse transcription PCR (qRT-PCR)}

All total RNA was extracted from BCa tissues or cells using the Trizol reagent (Invitrogen, Carlsbad, CA, USA). Qualified total RNA was then reverse transcribed to complementary DNA using a PrimeScript RT reagent Kit (Takara, Dalian, China). The quantitative real-time polymerase chain reaction (qRT-PCR) was performed using SYBR Green PCR kit (Takara, Dalian, China) following the manufacturer's instructions. GAPDH was measured as an internal control. The primer sequences were listed as follows: TMEM40 primers: (forward, 5'-GCGGTAGGGGTGTACGGT-3'; reverse, 5'-CCG GACACGCTGAACT TGT-3'); P53 primers: (forward, 
5-AAGATCCGCGGGCGTAA-3; reverse, 5'-CATCCT TTAACTCTAAG GCCTCATTC-3'); c-myc primers: (forward, 5'-CTTCTCTCCGTCCTCGGATTCT-3'; reverse, 5'-GAA GGTGATCCAGACTCTGACCTT-3'); JNK2 primers: (forward, $5^{\prime}$-TACGTGGTGACACGGTA CTACC-3'; reverse, 5'-CACAACCTTTCACCAGCTC TCC-3'); RB primers: (forward, 5'-ATCAAGGGTCATT ATGG GTTAGGC-3'; reverse, 5'-TAGGTGTAGGGGA GGGGAGAAGC-3'); GAPDH primers: (forward, $5^{\prime}$-CG CTCTCTGCTCCTCCTGTTC-3'; reverse, 5'-ATCCGT TGACTCCGACCTTCAC-3'). The reactions were carried out on an ABI PRISM 7500 Fluorescent Quantitative PCR System (Applied Biosystems, Foster City, CA, USA) in triplicate. The average value in each triplicate was used to calculate the relative amount of TMEM40 using $2^{-\Delta \Delta C t}$ methods.

\section{Western blot analysis and antibodies}

The $\mathrm{BCa}$ cells and tumor tissue samples lysates were extracted with RIPA cell lysis buffer, and the protein concentration in the lysates was quantified using an enhanced bicinchoninic acid protein assay kit (Thermo Fisher Scientific, MA, USA) with bovine serum albumin as a standard. The total protein extract will be used for western blot analysis. Equal amounts of total protein of tissues or cells were subjected to 10\% SDSPAGE and transferred to a PVDF membrane followed by immunoblotting using the following primary polyclonal antibodies: mouse anti-TMEM40 (Santa Cruz, CA, USA), rabbit anti-p53 (Proteintech Group, INC, USA), rabbit anti-p21 (Proteintech Group, INC, USA), mouse anti-CCND1 (Proteintech Group, INC, USA), rabbit anti-Caspase-3 (Cell Signaling Technology, MA, USA), rabbit anti-Caspase-9 (Proteintech Group, INC, USA), rabbit anti-PARP (Proteintech Group, INC, USA), rabbit anti-c-MYC (Proteintech Group, INC, USA) and GAPDH (Cell Signaling Technology, MA, USA). Specific proteins were detected with enhanced chemiluminescence (ECL, Millipore, USA). Band density was measured (ImageJ software) and normalized to GAPDH.

\section{Cell migration and invasion assay}

A scratch wound healing assay was adapted to evaluate the ability of cell migration. Cells at a density of $80-90 \%$ confluence in 12-well plates were scratched manually with a sterile $200 \mu \mathrm{L}$ plastic pipette tip, cultured for $24 \mathrm{~h}$ and photographed under a light microscope. The width of the wound area was quantitated, using the Image J. For the invasion assay, a transwell chamber was placed into a 24-well plate and was coated with $30 \mu \mathrm{L}$ Matrigel and incubated for $60 \mathrm{~min}$ at $37^{\circ} \mathrm{C}$. Cells were cultured in normal medium and transfected with corresponding siRNA or plasmid DNA. $24 \mathrm{~h}$ after transfection, $5 \times 10^{4}$ cells were first starved in $200 \mu \mathrm{L}$ serum free medium and then placed in the uncoated dishes. The lower chamber was filled with $750 \mu \mathrm{L}$ of complete medium. The cells were incubated for $48 \mathrm{~h}$ at $37^{\circ} \mathrm{C}$ in a $5 \% \mathrm{CO}_{2}$ incubator, and then the cells that had migrated to the bottom surface of the filter membrane were stained with $0.1 \%$ crystal violet staining solution and photographed in five preset fields per insert. The results represented the average of three independent experiments.

\section{Cell Counting Kit-8 assay}

Cell proliferation was determined using Cell Counting Kit-8 (Beyotime Inst Biotech, China). In short, $5 \times 10^{3}$ cells/well were seeded in a 96-well flat-bottomed plate for $24 \mathrm{~h}$, then transfected with corresponding siRNA or plasmid DNA cultured in normal medium. At 0, 24, 48 and $72 \mathrm{~h}$ after transfection, $10 \mu \mathrm{L}$ of CCK-8 $(5 \mathrm{mg} /$ $\mathrm{mL}$ ) was added to each well and the cells were cultured for $2 \mathrm{~h}$ then determined the absorbance at a wavelength of $450 \mathrm{~nm}$ using a microplate reader (Tecan, Infinite ${ }^{\circledR} \mathrm{M} 200$, Austria). Experiments were repeated at least three times.

\section{5-Ethynyl-2'-deoxyuridine (EdU) incorporation assay}

Cell proliferation was also determined by 5 -Ethynyl-2' deoxyuridine incorporation assay using an EdU Apollo DNA in vitro kit (RiboBio, Guangzhou, China) following the manufacturer's instructions. Briefly, 5637 and EJ cells were plated in confocol dish plates at a density of $4 \times 10^{3}-1 \times 10^{5}$ cells and incubated with $100 \mu \mathrm{L}$ of $50 \mu \mathrm{M}$ EdU per dish for $2 \mathrm{~h}$ at $37^{\circ} \mathrm{C}$, at $48 \mathrm{~h}$ after transfected with corresponding siRNA or plasmid DNA, respectively. Then, the cells were fixed for $30 \mathrm{~min}$ at room temperature using $100 \mu \mathrm{L}$ of fixing buffer ( $4 \%$ polyformaldehyde containing PBS). Subsequently, the cells were incubated with $100 \mu \mathrm{L}$ of $2 \mathrm{mg} / \mathrm{mL}$ glycine for $5 \mathrm{~min}$ followed by washing with $100 \mu \mathrm{L}$ of PBS (Phosphate Buffered Saline). After permeabilization with $100 \mu \mathrm{L}$ of $0.5 \%$ Triton X-100 for $10 \mathrm{~min}$, the cells were reacted 
with $100 \mu \mathrm{L}$ of $1 \times$ Apollo solution for $30 \mathrm{~min}$ at room temperature in the dark. After that, cells were incubated with $100 \mu \mathrm{L}$ of $1 \times$ Hoechst 33342 solution for $30 \mathrm{~min}$ at room temperature in the dark followed by washing with $100 \mu \mathrm{L}$ of PBS. The cells were then visualized under a fluorescence microscopy. Experiments were repeated at least three times.

\section{Cell cycle assay}

The $\mathrm{BCa}$ cells were plated in 6-well plates at a density of $1 \times 10^{6}$ cells every well cultured for $24 \mathrm{~h}$, then transfected with siRNA and plasmid DNA. After $48 \mathrm{~h}$, cells were then treated with trypsin, fixed in 70\% frozen ethanol, followed by overnight incubation and washed with PBS, nucleus was stained by $450 \mu \mathrm{L}$ Propidium Iodide (PI) and $50 \mu \mathrm{L}$ RNAase. The percentages of 5637/EJ cells in the G0/G1, S, and G2/M phases were examined by Becton-Dickinson FACSVerse ${ }^{\mathrm{TM}}$ flow cytometry (San Jose, CA, USA). All experiments were performed three times.

\section{Cell apoptosis assay}

Briefly, $\mathrm{BCa}$ cells were plated in 6-well plates at a density of $1-5 \times 10^{5}$ cells every well cultured for $24 \mathrm{~h}$. After transfection for $48 \mathrm{~h}$, cells were harvested by trypsinization and washed with PBS and suspended in $500 \mu \mathrm{L}$ Annexin $V$ binding buffer. The percentage of cells actively undergoing apoptosis was determined by double staining with $5 \mu \mathrm{L}$ AnnexinV-APC and $5 \mu \mathrm{L}$ 7-AAD apoptosis detection kits (KeyGEN Biotech, Nanjing, China), and analyzed using a Becton-Dickinson FACSVerse ${ }^{\mathrm{TM}}$ flow cytometry (San Jose, CA, USA). At least 10,000 cells were obtained from each sample.

\section{Tumorigenicity assay in nude mice}

Five-week-old female/male BALB/c-nu athymic nude mice (Experimental Animal Center of Southern Medical University, Guangzhou, China) were subcutaneously injected in the right flank with $1.0 \times 10^{7}$ cells in $0.2 \mathrm{~mL}$ of PBS. Once tumors were formed, tumor volume (V) was measured daily by caliper and calculated using the formula $\mathrm{V}=\left(\mathrm{L} \times \mathrm{W}^{2}\right) / 2$, where $\mathrm{L}$ was the length and $\mathrm{W}$ was the width of the tumor. The mice were randomly divided into two groups $(\mathrm{n}=6)$ for inoculation of shRNA transfected EJ cells and negative control (NC) for 27 days. Growth curves were plotted using average tumor volume within each experimental group every 3 days. 4 weeks later, the mice were euthanized, and the dissected tumors were collected. All animal experiments were approved by the animal center of the Southern Medical University.

\section{Statistical analysis}

All statistical analyses were performed using the IBM SPSS 20.0 software package (SPSS, Inc., Chicago, IL, USA). Group difference was assessed using Student's $t$ test. Data are presented as the mean \pm SD based on at least three repeats. Differences were considered to be statistically significant at $\mathrm{P}<0.05$.

\section{Results}

Expression of TMEM40 was upregulated in BCa tissues and cell lines compared with their normal entities

From the expression of TMEM40 RNAseq data of the Cancer Genome Atlas (TCGA), we found that TMEM40 expression levels was significantly increased in $\mathrm{BCa}$ and other organ cancers compared with their controls (Fig. 1a, $\mathrm{N}=30$ ). Furthermore, from the Gene Expression Profiling Interactive Analysis (GEPIA), we also found that the level of TMEM40 in the tumor tissues $(\mathrm{N}=404)$ was significantly higher than that in the controlled bladder tissues $(\mathrm{N}=28$ ) (Fig. $1 \mathrm{~b}, P<0.05)$. To investigate the expression status of TMEM40 in $\mathrm{BCa}$, western blotting and quantitative RT-PCR analyses were performed in seven BCa cell lines (i.e. EJ, T24, 5637, SW780, UMUC3, J82 and BIU-87), one normal uroepithelial cell line SV-HUC-1 and 12 cases fresh BCa tissues paired with their adjacent non-neoplastic bladder tissues. The results showed that TMEM40 were significantly upregulated in five BCa cell lines (i.e. EJ, T24, 5637, UMUC3 and J82) compared with normal uroepithelial cell line SV-HUC-1 both on protein and mRNA

\footnotetext{
(See figure on next page.)

Fig. 1 TMEM40 was overexpressed in bladder cancer tissues and cancer cells. a TMEM40 mRNA expression was significantly increased in bladder cancer and other organ cancers compared with their controls $(n=30)$. b Quantification of TMEM40 mRNA expression in bladder cancer and normal tissues. TMEM40 expression was significantly increased in bladder cancer tissues $(n=404)$ compared with normal tissues from the patients $(n=28)$ from the GEPIA dataset. Differences in TMEM40 expression levels between bladder cancer tissues and pair-matched noncancerous tissues. The expression of TMEM40 was normalized to that of GADPH. Statistical differences between samples were analyzed with paired samples t-test $(n=12)$. d Protein. $\mathbf{f}$ mRNA. Expression level of TMEM40 in seven BC cell lines T24, 5637, EJ, J82, UMUC3, SW780, BIU87 and normal urothelial cell line SVHUC-1 cells. c Protein. e mRNA. Data are presented as mean \pm SD based on at least three independent experiments $\left({ }^{*} P<0.05,{ }^{* *} P<0.01\right.$, ***P $<0.001)$
} 


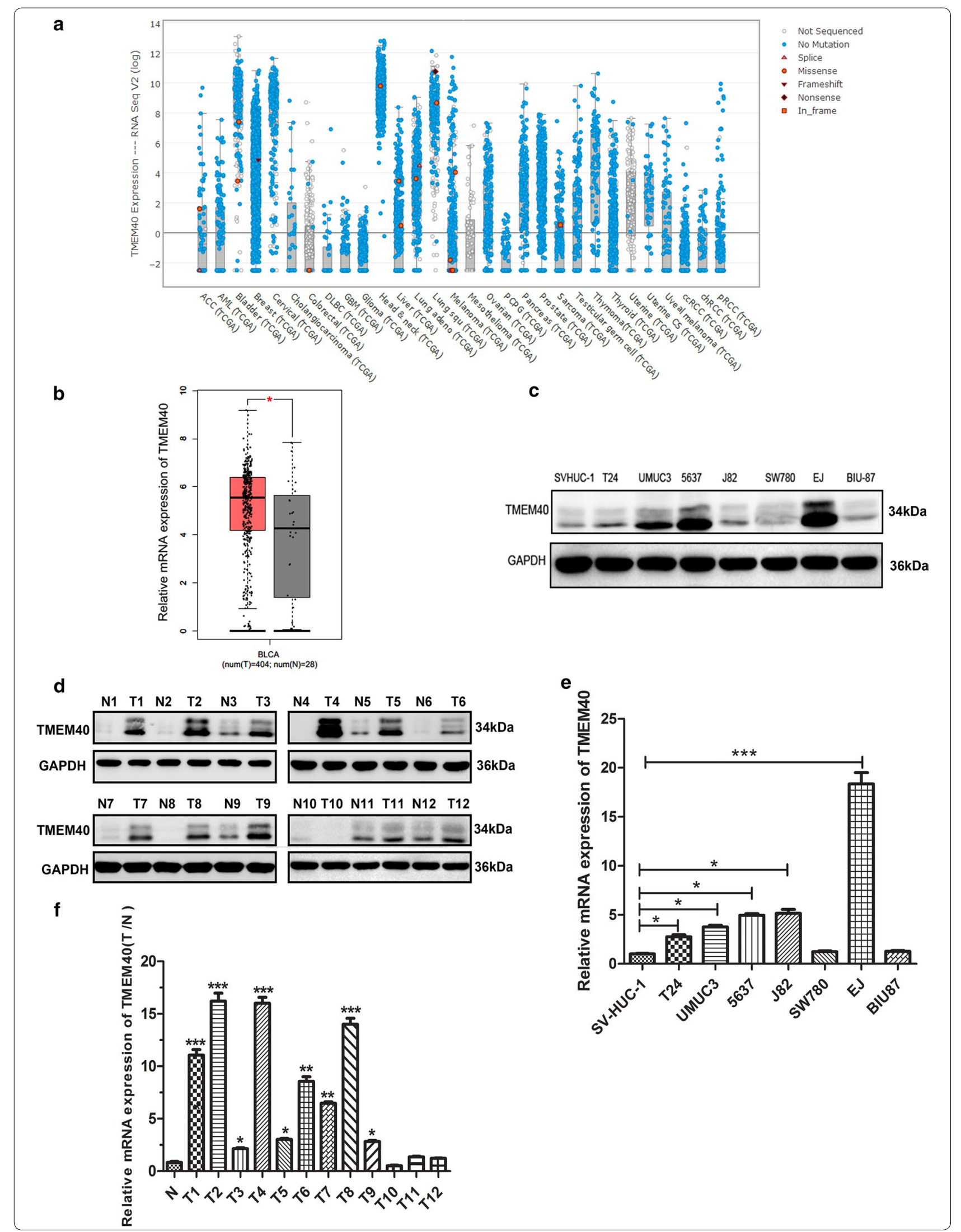


levels (Fig. 1c, e, $P<0.05$ ). Similarly, TMEM40 were considerably higher in $\mathrm{BCa}$ tissue specimens when compared with their paired normal bladder tissues (Fig. 1d, f, $P<0.05)$.

\section{TMEM40 knockdown in BCa cells transfected with siRNA against TMEM40 or it upregulation by $\mathrm{pEZ-M98}$ vector} To investigate the functions of TMEM40 in $\mathrm{BCa}$, overexpression vector containing green fluorescence protein (GFP) (Fig. 2a) were used to increase the expression of TMEM40 in 5637 and EJ cells. Then transfected with the vector and tested the efficiency of fluorescence expression (Fig. 2b). The data showed that pEZ-M98 vector could upregulate both TMEM40 mRNA (Fig. 2e, f) and protein (Fig. 2c) expressions in BCa cells. Meanwhile, we inhibited TMEM40 expression in BCa cells 5637 and EJ by transfecting them with its specific siRNA. Then we performed qRT-PCR and western blot to measure its expression levels at $48 \mathrm{~h}$ post-transfection. The data showed that TMEM40 expression could be effectively suppressed in BCa cells (Fig. 2d, g, h, $P<0.05$ ).

\section{Silencing TMEM40 suppressed cell proliferation, migration and invasion, promoted cell apoptosis in BCa cells}

Based on the aforementioned results, we further evaluated whether TMEM40 could affect the biologic activities of BCa cells. Wound healing assay was used to assess the capability of cell motility, and the results showed that 5637 and EJ cells transfected with siRNA underwent a slower closing of scratch wounds compared with the negative control groups (Fig. 3a, b, $P=0.001 ; P=0.002$ ). Then transwell assay was applied to evaluate its impact on invasion ability in $\mathrm{BCa}$ cells, and the findings indicated that silencing TMEM40 significantly repressed the capability of invasion of BCa cells (Fig. 3c, d, $P<0.001$ ). Meanwhile, the Cell Counting Kit-8 (CCK-8) (Fig. 4a-d, $P<0.05$ ) and 5-Ethynyl-2'-deoxyuridine (EdU) (Fig. 4e$\mathrm{h}, \mathrm{P}<0.05)$ assays demonstrated that suppression of TMEM40 attenuated cell proliferation in 5637 and EJ cells. Furthermore, results from the cell cycle analysis suggested that TMEM40 knockdown induced G1 phase arrest both in 5637 and EJ cells (Fig. 5a, b, $P<0.05$ ). Finally, the effect of TMEM40 on cellular apoptosis was explored. FACS analysis showed that there was a significantly higher percentage of Annexin V-positive cells in TMEM40-silenced 5637 and EJ cells compared with control cells (Fig. 5c, d, $P<0.01$ ). This indicated that TMEM40 knockdown induced cellular apoptosis. In contrast, overexpressing TMEM40 had proliferation, migration and invasion promoting effect as determined by the wound healing, CCK-8, EdU and transwell assay (Figs. 3, 4, 5, $P<0.05$ ). From these results, we speculated that TMEM40 may play some important roles in $\mathrm{BCa}$ cells.

\section{Effects of TMEM40 on tumor growth in vivo and the expression of oncogenes and tumor suppressor genes} The short hairpin RNA (shRNA) (Fig. 6a) was introduced into 5637 and EJ cell lines, which exhibit low TMEM40 expression, whereas exogenous TMEM40 was stably introduced into 5637 cell, which shows relative high TMEM40 expression (Fig. $6 \mathrm{~b}-\mathrm{d}, P<0.05$ ). To confirm the oncogenic efficiency of TMEM40 in vivo, we constructed a BALB/c nude mouse xenograft model by EJ cells. Our results demonstrated the tumor weight and volume of tumors in nude mice treated with shRNA were markedly suppressed $(38.5 \%$ of decrease in tumor weight) relative to that of treated with empty vector (Fig. 6f, $P<0.05$ ). These data indicated that knockdown of TMEM40 markedly inhibited the tumorigenicity of EJ cells in the nude mouse xenograft model (Fig. 6e, g, $P<0.05)$. Moreover, we checked the expression levels of oncogenes (i.e. c-myc), as well as tumor suppressor genes (i.e. p53, Rb and JNK2), all of which were well known to be involved in urothelial tumorigenesis, in $\mathrm{NC}$ versus siRNA cells. TMEM40 silencing resulted in significant downregulation of the expression of c-myc as well as significant upregulation of the expression of $\mathrm{p} 53, \mathrm{Rb}$ and JNK2 (Fig. 7a, $P<0.05$ ).

(See figure on next page.)

Fig. 2 pEZ-M98-TMEM40-GFP vector construction and the expression of TMEM40. a The overexpression vector pEZ-M98-TMEM40 was engineered to express TMEM40 under the T7 promoter. $\mathbf{b}$ The expression of green fluorescent protein after $48 \mathrm{~h}$ of pEZ-M98 vector/empty vector transfection in 5637 and EJ cells (magnification $\times 100$ ). The pEZ-M98 significantly upregulated the expression level of TMEM40 in 5637 and EJ cells. c Protein. e, $\mathbf{f}$ mRNA. The TMEM40-siRNA significantly down-regulated the expression level of TMEM40 in 5637 and EJ cells. $\mathbf{d}$ Protein. $\mathbf{g}, \mathbf{h}$ mRNA $\left({ }^{*} P<0.05\right.$, ${ }^{*} P<0.01$, ${ }^{* *} P<0.001$ ) 


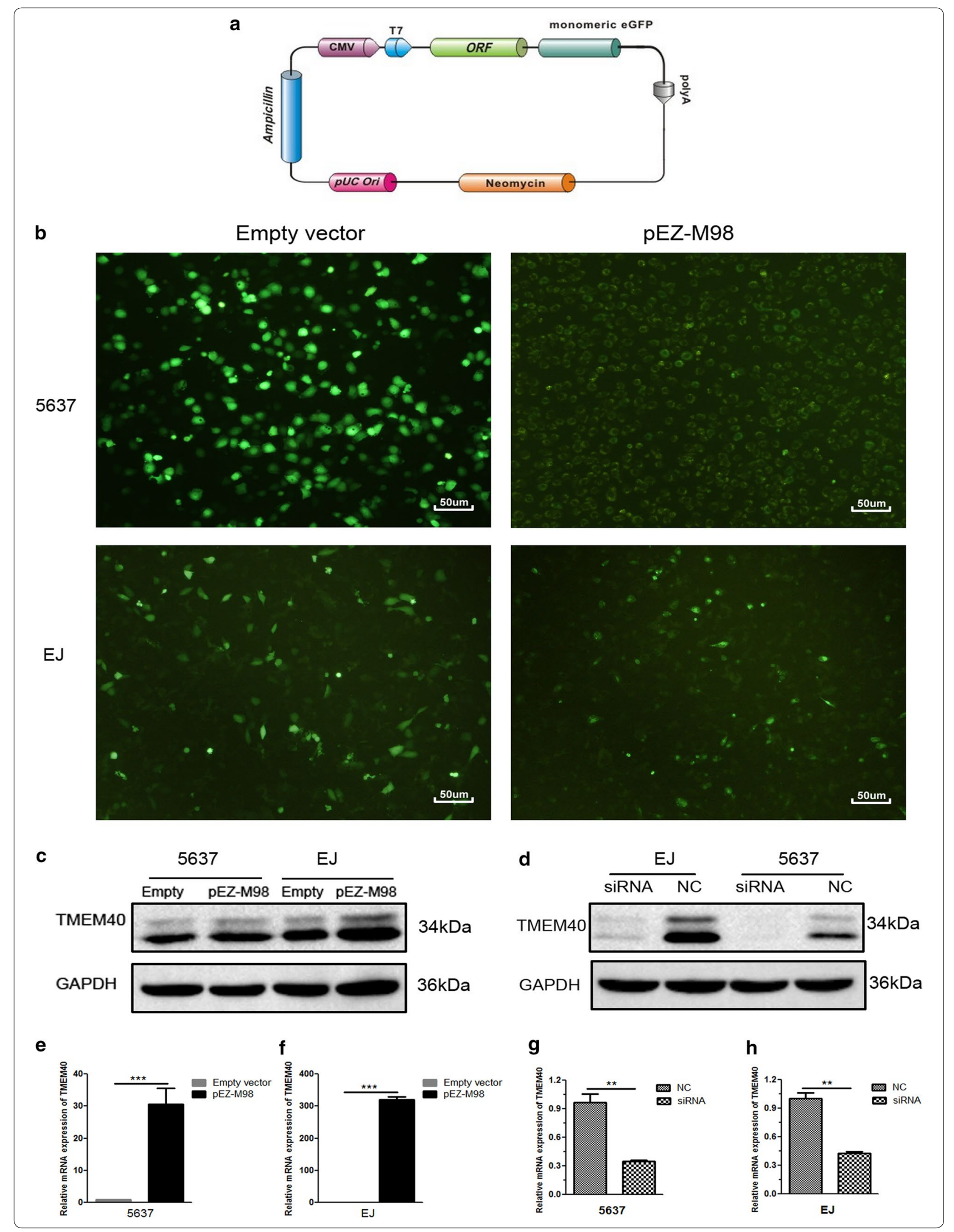


a

EJ

$24 h$

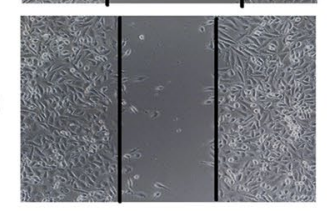

b

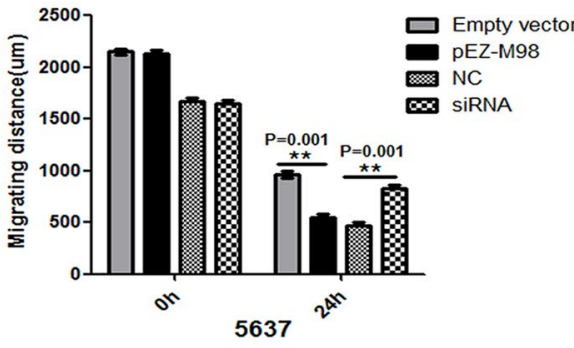

c

5637

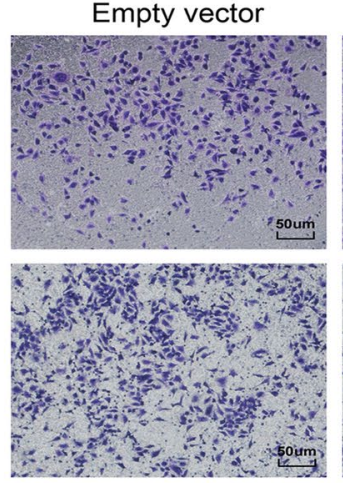

pEZ-M98
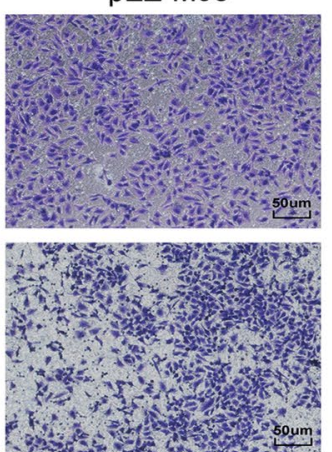
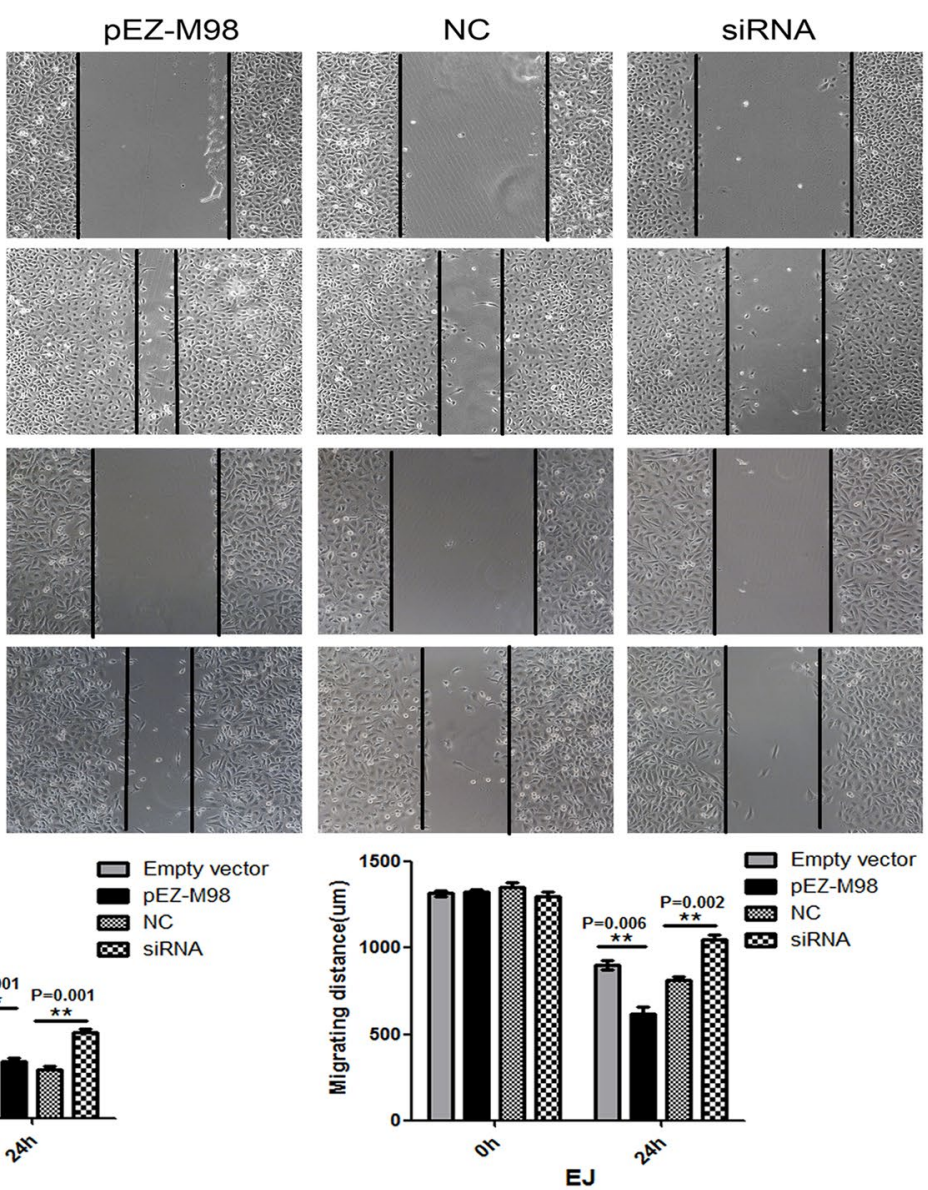

NC

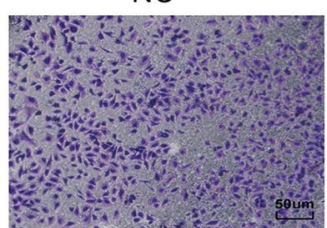

SiRNA
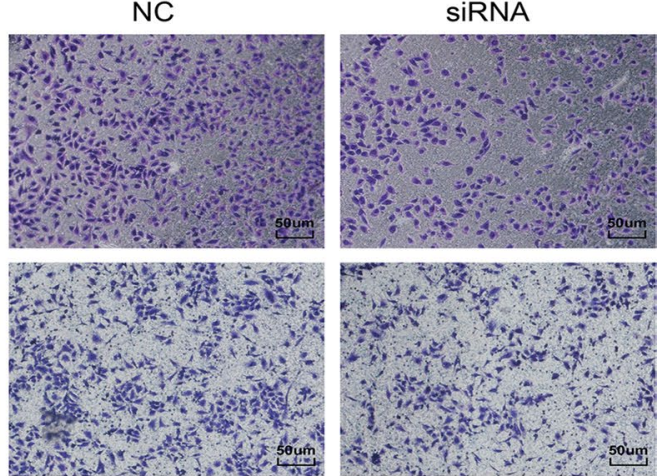

d

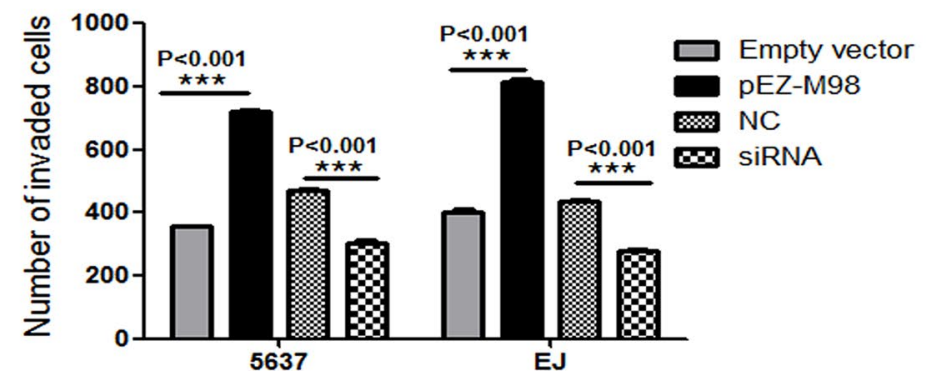

Fig. 3 Overexpression of TMEM40 promotes bladder cancer cell migration and invasion. Cell migration and invasion was determined by wound healing and transwell assay. $\mathbf{a}$, b Wound healing assay showed that TMEM40 shRNA resulted in a slower closing of scratch wounds was observed in bladder cancer 5637 and EJ cells. c, d Transwell invasion assay was measured and the results were expressed as the number of invaded cells per field compared with respective control. Data are expressed as the mean $\pm \mathrm{SD}$ (magnification $\times 100,{ }^{*} P<0.05,{ }^{* *} P<0.01,{ }^{* * *} P<0.001$ ) 

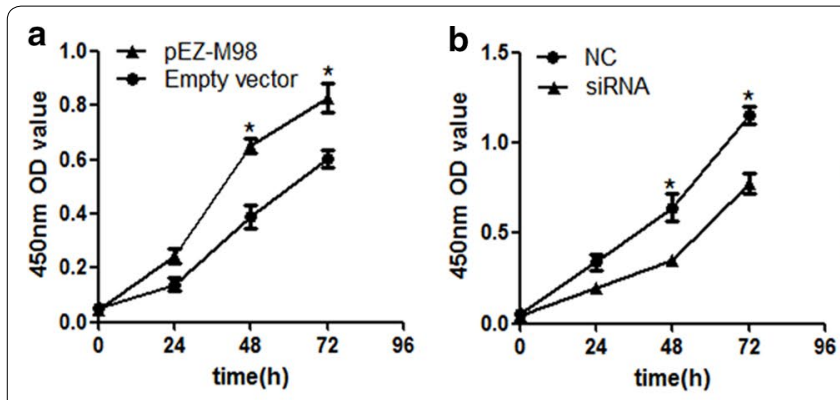

5637
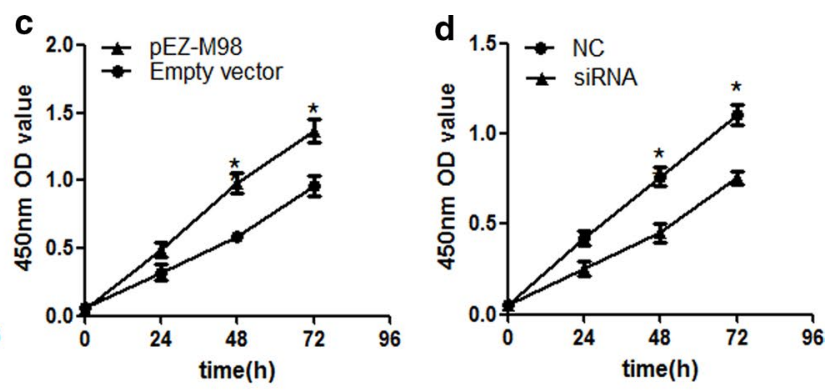

EJ e
Empty vector
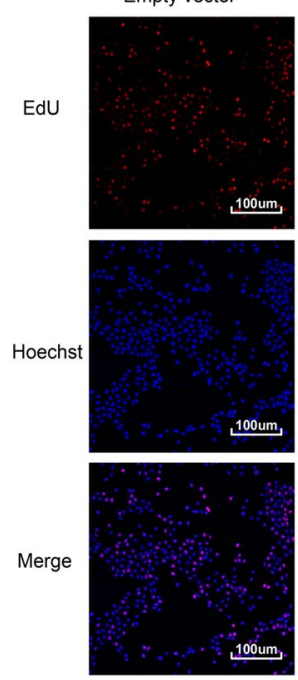

$100 \mathrm{um}$
pEZ-M98
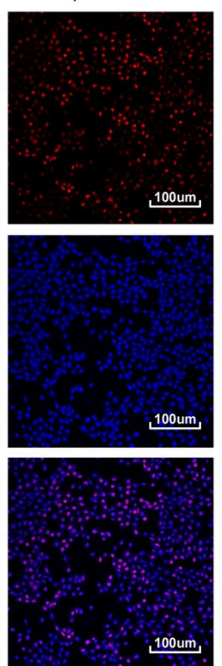

NC
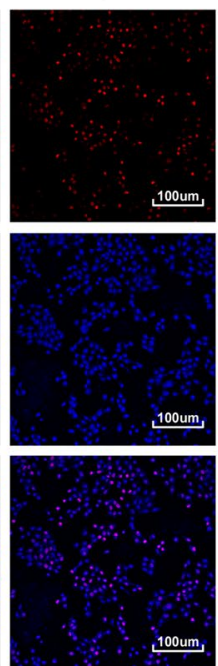

5637

g

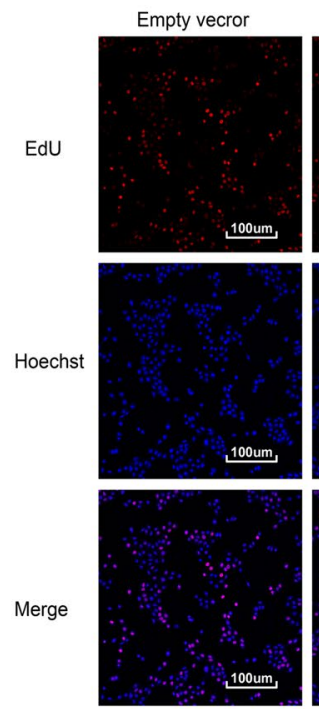

pEZ-M98
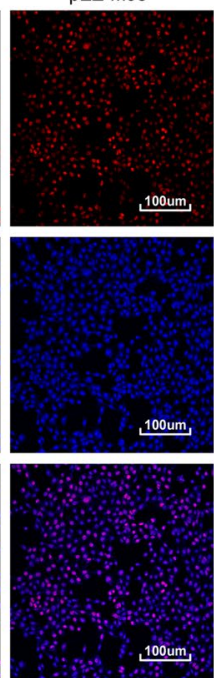

NC
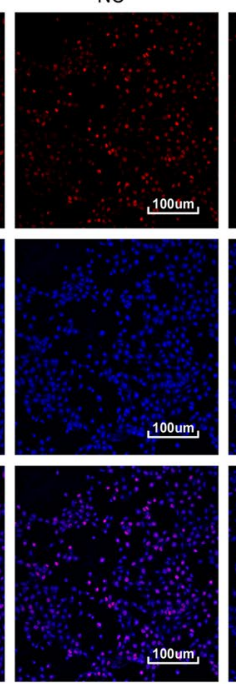

siRNA
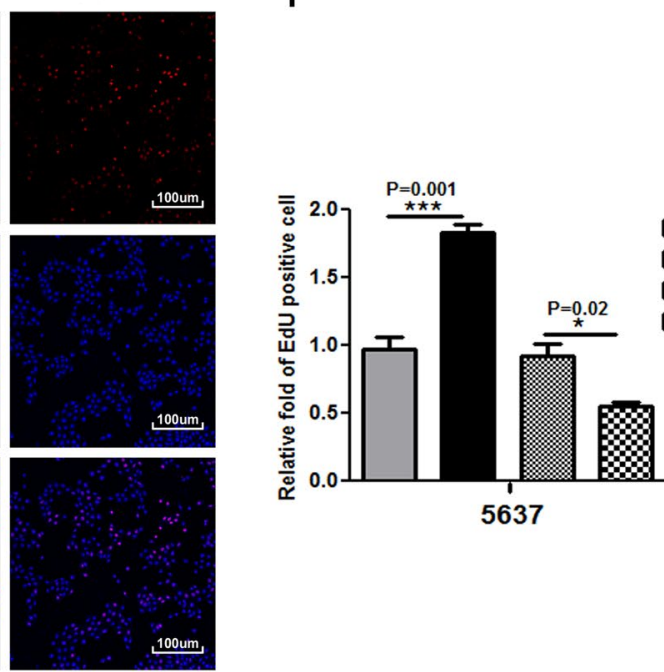

SiRNA
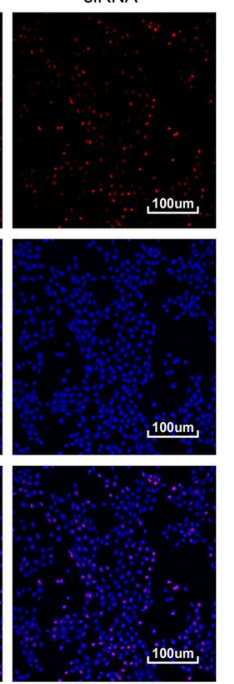

EJ

f

Empty vector

pEZ-M98

\& $N C$

$\mathbf{0}$ siRnA

h

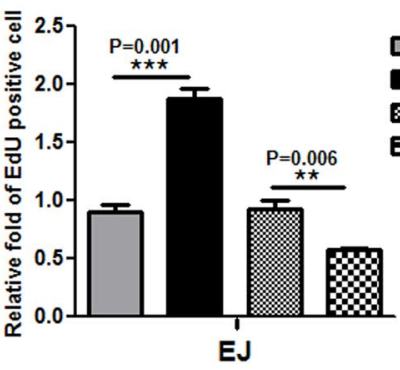

Fig. 4 Effects of upregulation or downregulation of TMEM40 on cell proliferation in bladder cancer cells. Cell proliferation was determined by CCK-8 and EdU assay. Cell proliferation promotion was observed in bladder cancer 5637 cells (a), EJ cells (c). Cell proliferation inhibition was observed in bladder cancer 5637 cells (b), EJ cells (d). Whereas TMEM40 overexpression promote $5637(\mathbf{e}, \mathbf{f})$ and EJ cells $(\mathbf{g}, \mathbf{h})$ proliferation. Data are shown as mean $\pm \mathrm{SD}$ (magnification $\times 100,{ }^{*} P<0.05,{ }^{* *} P<0.01$, ${ }^{* *} P<0.001$ ) 


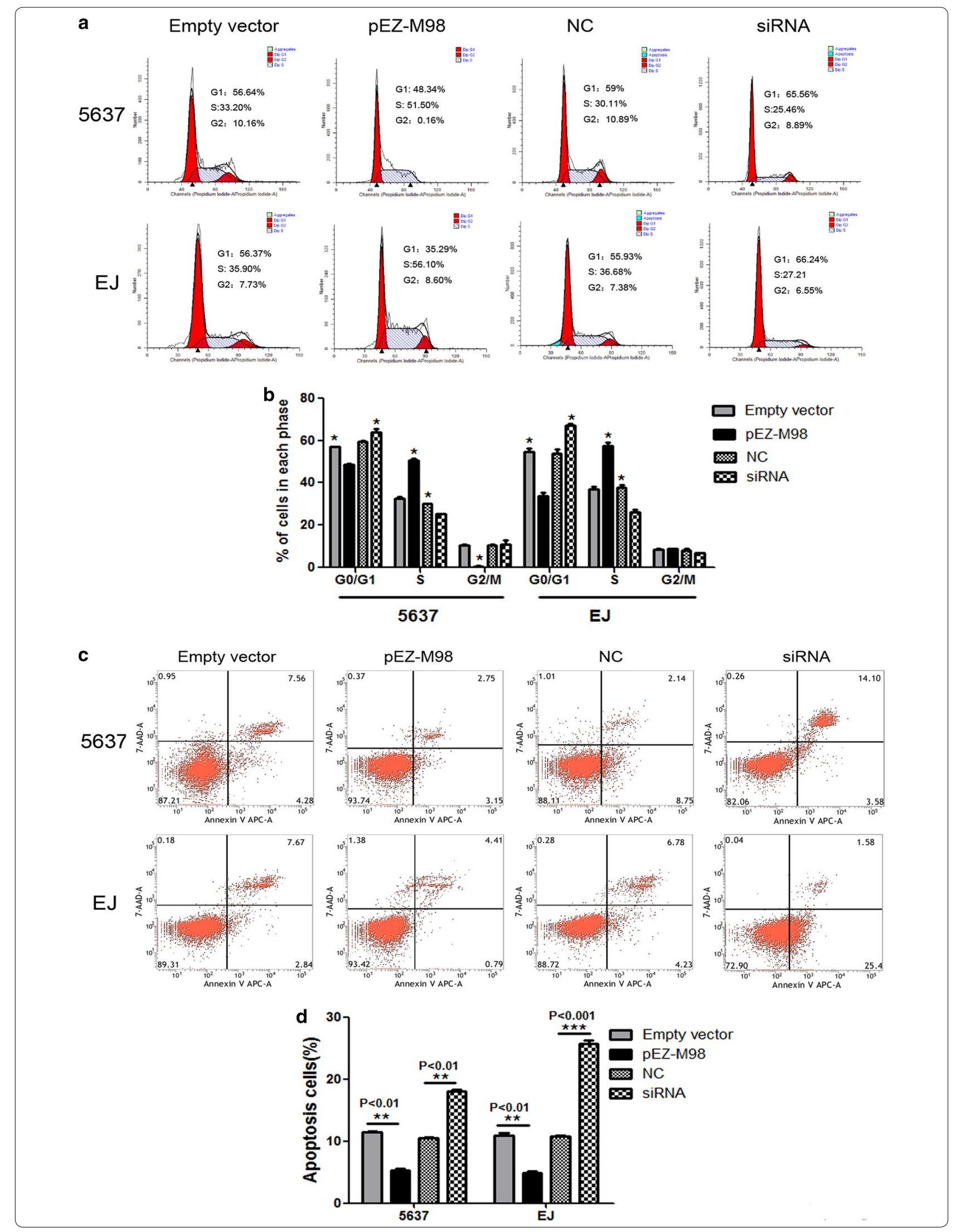


(See figure on previous page.)

Fig. 5 Overexpression of TMEM40 induced apoptosis and S phase cell cycle arrest in 5637 and EJ cells. Cell apoptosis and cell cycle was determined by flow cytometry. $\mathbf{a}, \mathbf{b}$ Cell cycle analysis determined the relative cell numbers in each cell-cycle phase after propidium iodide staining of pEZ-M98 vector or siRNA 5637 and EJ cells. c, d Apoptotic cells increased significantly after $48 \mathrm{~h}$ transfection with siRNA compared to controls. Data represent the mean $\pm \mathrm{SD}$ from three independent experiments $\left({ }^{*} P<0.05,{ }^{* *} P<0.01,{ }^{* * *} P<0.001\right.$ )

\section{TMEM40 acted as an oncogene via regulating the p53}

\section{pathway}

To comprehensively find mechanistic insight into the role of TMEM40 in regulating $\mathrm{BCa}$ cell proliferation and apoptosis, we measured the alteration of proliferation-related signals (p53, p21, c-MYC, cyclin D1) and apoptosis-related proteins ( $\mathrm{p} 53$, activated caspase-3, activated caspase-9, activated PARP) by western blotting. Knockdown of TMEM40 increased the expression levels of p53 and p21, activated caspase-3, caspase-9, and PRAP and decreased the levels of c-MYC and cyclin D1 in 5637 and EJ cells. Together, all the above suggested that TMEM40 knockdown reduced the proliferation and enhanced apoptosis of BCa cells (Fig. 7b, c, $P<0.05)$.

\section{Discussion}

$\mathrm{BCa}$ is one of the most common urinary malignancies worldwide [14]. Most patients are diagnosed at advanced stage of $\mathrm{BCa}$ because patients with $\mathrm{BCa}$ are lack of specific symptoms at early stage [15-18]. Nevertheless, the poor understanding of $\mathrm{BCa}$ development mechanism has limited the effective adjuvant therapies for $\mathrm{BCa}[19-21]$.

To the best of our knowledge, the biological functions of TMEM40 in BCa have not been characterized yet. In the present study, we found that TMEM40 expression was significantly up-regulated in $\mathrm{BCa}$ tissues and cells compared with non-neoplastic urothelium. We identified that TMEM40 overexpression is a characteristic molecular change in $\mathrm{BCa}$ and explore the biological roles of TMEM40 on the phenotypes of BCa cells in vitro. To investigate the TMEM40 biological functions in $\mathrm{BCa}$, we used CCK8, EdU, scratch assays, transwell and apoptosis assays to detect cell proliferation, migration, invasion and apoptosis, respectively. TMEM40 silencing results in inhibition of $\mathrm{BCa}$ cell proliferation, migration, invasion and induced cell apoptosis. Furthermore, TMEM40 knockdown also blocked G1-to-S cell cycle transition and these effects could be reversed by overexpressed it in $\mathrm{BCa}$ cells.

In mouse xenograft models for BCa, TMEM40 knockdown was also found to considerably retard tumor formation and subsequent tumor growth [22]. Moreover, TMEM40 knockdown was associated with downregulated expression of oncogenes (i.e. c-myc) and upregulated expression of tumor suppressor genes (i.e. p53, Rb and JNK2) in BCa cells [23]. These findings in conjunction with our in vitro data suggested that TMEM40 played important roles in the malignant phenotype regulation of $\mathrm{BCa}$ and likely to promote bladder tumorigenesis and tumor progression.

The tumor suppressor p53 is a transcription factor that regulates several cellular stress responses, including DNA repair, metabolism, cell cycle arrest, genomic stability, apoptosis and senescence, through induction of the various transcriptions of target genes [24-26]. We finally investigated the levels of proliferation-related signals (i.e. p53, p21, c-MYC, cyclin D1) and apoptosisrelated proteins (i.e. $\mathrm{p} 53$, activated caspase-3, activated caspase-9, activated PARP) by western blotting. Our results revealed that knockdown of TMEM40 increased the levels of p53, p21, activated caspase-3, caspase-9, and PRAP and decreased the levels of c-MYC, cyclin D1 in 5637 and EJ cells [27-29]. These findings suggest that TMEM40 was a proto-oncogene which could promote $\mathrm{BCa}$ development and progression via the p53 signaling pathway in $\mathrm{BCa}$.

\section{Conclusions}

Taken together, our findings provide evidence that TMEM40 play oncogenic roles in the development and progression of $\mathrm{BCa}$ through the $\mathrm{p} 53$ signaling pathway. And our data gives a more comprehensive understanding of how TMEM40 functions in $\mathrm{BCa}$ and demonstrates its potential as a powerful therapeutic approach to treat $\mathrm{BCa}$. 


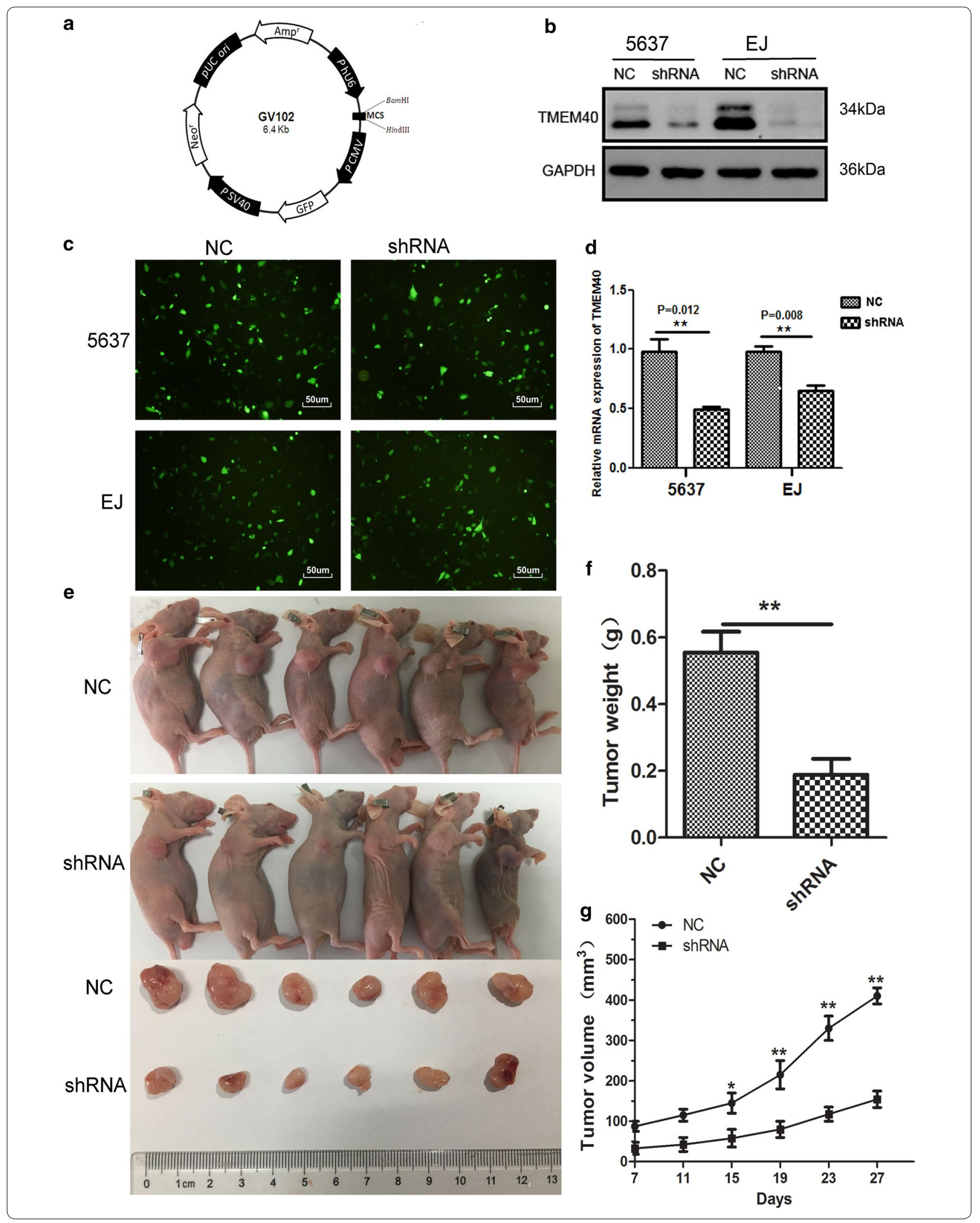


(See figure on previous page.)

Fig. 6 TMEM40 shRNA suppresses the expression of TMEM40 in bladder cancer cells in vitro and tumorigenicity in vivo. a The knockdown vector hU6-MCS-CMV-GFP-SV40-Neomycin was engineered to express shRNA under the U6 promoter. c The expression of green fluorescent protein after $48 \mathrm{~h}$ of shRNA vector/NC vector transfection in 5637 and EJ cells (magnification $\times 100$ ). The shRNA significantly downregulated the expression level of TMEM40 in 5637 and EJ cells. b Protein. d mRNA. e Representative images of tumorigenicity assay performed in nude mice. f Tumor weight was measured after sacrifice at the end of the experiment. $\mathbf{g}$ Tumor volume was monitored by caliper measurements twice weekly. All results were presented as the mean \pm SD from 3 independent experiments $\left({ }^{*} P<0.05,{ }^{* *} P<0.01,{ }^{* * *} P<0.001\right)$

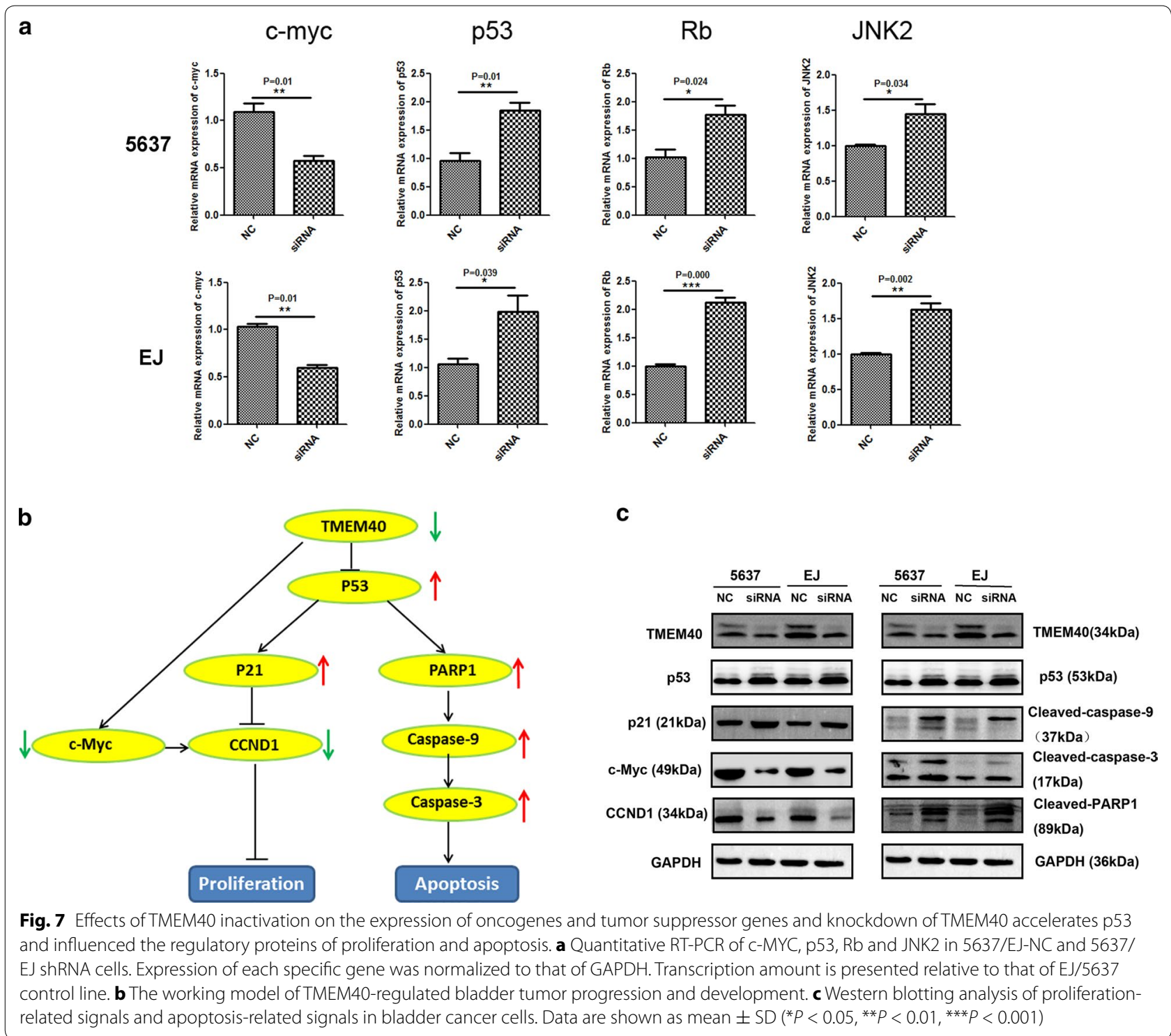

\section{Abbreviations}

TMEM40: transmembrane protein 40; BCa: bladder cancer; FBS: fetal bovine serum; GRN: granulin; DMEM/F12: Dulbecco's Modified Eagle Medium/Nutrient Mixture F-12; qRT-PCR: quantitative real-time PCR; PVDF: polyvinylidene fluoride; CCK-8: Cell Counting Kit-8; EdU: 5-ethynyl-2'-deoxyuridine; NC: negative control; siRNA: small interfering RNA; SDS-PAGE: sodium dodecyl sulfate polyacrylamide gel electrophoresis; shRNA: short hairpin RNA; RPMI-1640: Roswell Park Memorial Institute 1640; FTLD: frontotemporal lobar degeneration; CIA: collagen induced arthritis; PARP: poly(ADP-ribose) polymerase;
GAPDH: glyceraldehyde-3-phosphate dehydrogenase; GFP: green fluorescence protein; PBS: phosphate buffered saline.

\section{Authors' contributions}

$J Z$ and RS conceived the study, participated in its design and coordination and helped to draft the manuscript. ZZ, QZ and HZ performed the experiments. ZZ and $S L$ coordinated the research and analyzed the data. $Z Z$ and $Y Z$ supported the experiments and helped to draft the manuscript. YF and SZ performed the 
statistical analysis. ZZ wrote the manuscript. All authors read and approved the final manuscript.

\section{Author details}

${ }^{1}$ Department of Biochemistry and Molecular Biology, School of Basic Medical Sciences, Southern Medical University, Guangzhou 510515, People's Republic of China. ${ }^{2}$ Guangdong Provincial Key Laboratory for Biochip Technology, Guangzhou 510515, People's Republic of China. ${ }^{3}$ The First School of Clinical Medicine, Southern Medical University, Guangzhou, People's Republic of China. ${ }^{4}$ Department of Biology Medicine and Advanced Materials Research Center, Shantou University, Shantou 515063, Guangdong, People's Republic of China.

\section{Acknowledgements}

We sincerely thank other students in our laboratory for their active help in this study.

\section{Competing interests}

The authors declared that they have no competing interests.

\section{Availability of data and materials}

All data and materials are well documented.

\section{Consent for publication}

Not applicable.

\section{Ethics approval and consent to participate}

All the patients signed informed consent and the study was approved by the Ethics Committee of Nanfang Hospital of Southern Medical University. All animal experiments were approved by the animal center of the Southern Medical University.

\section{Funding}

The present study was supported by the National Natural Science Foundation of China $(81372154,81672588)$ and the Teaching and Research Award Program for Outstanding Young Teachers in Higher Education institutions of Guangdong Province, China (2014).

\section{Publisher's Note}

Springer Nature remains neutral with regard to jurisdictional claims in published maps and institutional affiliations.

Received: 20 October 2017 Accepted: 28 December 2017 Published online: 19 January 2018

\section{References}

1. Torre LA, Bray F, Siegel RL, Ferlay J, Lortet-Tieulent J, Jemal A. Global cancer statistics, 2012. CA Cancer J Clin. 2015;65:87-108.

2. Huang P, Chen J, Wang L, Na Y, Kaku H, Ueki H, Sasaki K, Yamaguchi K, Zhang K, Saika T, et al. Implications of transcriptional factor, OCT-4, in human bladder malignancy and tumor recurrence. Med Oncol. 2012;29:829-34

3. Ferlay J, Shin H, Bray F, Forman D, Mathers C, Parkin DM. Estimates of worldwide burden of cancer in 2008: GLOBOCAN 2008. Int J Cancer. 2010;127:2893-917.

4. Cambier S, Sylvester RJ, Collette L, Gontero P, Brausi MA, van Andel G, Kirkels WJ, Silva FCD, Oosterlinck W, Prescott S, et al. EORTC nomograms and risk groups for predicting recurrence, progression, and disease-specific and overall survival in non-muscle-invasive stage Ta-T1 urothelial bladder cancer patients treated with 1-3 years of maintenance Bacillus CalmetteGuérin. Eur Urol. 2016;69:60-9.

5. Suer E, Hamidi N, Gokce MI, Gulpinar O, Turkolmez K, Beduk Y, Baltaci S. Significance of second transurethral resection on patient outcomes in muscle-invasive bladder cancer patients treated with bladder-preserving multimodal therapy. World J Urol. 2016;34:847-51.

6. Yue $Y$, Grossmann B, Ferguson-Smith M, Yang F, Haaf T. Comparative cytogenetics of human chromosome 3 q21.3 reveals a hot spot for ectopic recombination in hominoid evolution. Genomics. 2005:85:36-47.
7. Yue Y, Grossmann B, Tsend-Ayush E, Grützner F, Ferguson-Smith MA, Yang F, Haaf T. Genomic structure and paralogous regions of the inversion breakpoint occurring between human chromosome 3p12.3 and orangutan chromosome 2. Cytogenet Genome Res. 2004;108:98-105.

8. Muller S, Stanyon R, Finelli P, Archidiacono N, Wienberg J. Molecular cytogenetic dissection of human chromosomes 3 and 21 evolution. Proc Natl Acad Sci USA. 2000;97:206-11.

9. Braga EA, Kashuba VI, Maliukova AV, Loginov VI, Senchenko VN, Bazov IV, Kiselev LL, Zabarovskii ER. New tumor suppressor genes in hot spots of human chromosome 3: new methods of identification. Mol Biol (Mosk). 2003:37:194-211.

10. Darai E, Kost-Alimova M, Kiss H, Kansoul H, Klein G, Imreh S. Evolutionarily plastic regions at human 3 p21.3 coincide with tumor breakpoints identified by the "elimination test". Genomics. 2005;86:1-12.

11. Yu X, Teng H, Marques A, Ashgari F, Ibrahim SM. High resolution mapping of Cia3: a common arthritis quantitative trait loci in different species. J Immunol. 2009;182:3016-23.

12. Milanesi E, Bonvicini C, Alberici A, Pilotto A, Cattane N, Premi E, Gazzina S, Archetti S, Gasparotti R, Cancelli V, et al. Molecular signature of disease onset in Granulin mutation carriers: a gene expression analysis study. Neurobiol Aging. 2013;34:1837-45.

13. Zhang QY, Fu MT, Zhang ZF, Feng YZ, Wei M, Zhou JY, Shi R. Expression of TMEM40 in bladder cancer and its correlation with clinicopathological parameters. Int J Clin Exp Pathol. 2017;10:8050-7.

14. $X u X L$, Li SQ, Lin YW, Chen H, Hu ZH, Mao YQ, Xu X, Wu J, Zhu Y, Zheng XY, Luo JD, Xie LP. MicroRNA-124-3p inhibits cell migration and invasion in bladder cancer cells by targeting ROCK1. J Transl Med. 2013;11:276.

15. Liu L, Qiu MN, Tan GB, Liang ZJ, Qin Y, Chen LQ, Chen HG, Liu JJ. miR-200C Inhibits invasion, migration and proliferation of bladder cancer cells through down-regulation of BMI-1 and E2F3. J Transl Med. 2014;12:305.

16. Wang JP, Jiao Y, Wang $C Y, X u Z B$, Zhang B. Rb knockdown accelerates bladder cancer progression through E2F3 activation. Int J Oncol. 2017;50:149-60.

17. Marta GN, Hanna SA, Gadia R, Correa SF, Silva UL, Carvalho HA The role of radiotherapy in urinary bladder cancer: current status. Int Braz J Urol. 2012;38:144-53.

18. Racioppi M, D'Agostino D, Totaro A, Pinto F, Sacco E, D'Addessi A, Marangi F, Palermo G, Bassi PF. Value of current chemotherapy and surgery in advanced and metastatic bladder cancer. Urol Int. 2012:88:249-58.

19. Vousden $\mathrm{KH}$, Prives $\mathrm{C}$. Blinded by the light: the growing complexity of p53. Cell. 2009:137:413-31.

20. Lin HY, Huang $\mathrm{CH}$, Wu WJ, Chou YH, Fan PL, Lung FW. Mutation of the p53 tumor suppressor gene in transitional cell carcinoma of the urinary tract in Taiwan. Kaohsiung J Med Sci. 2005;21:57-64.

21. Chen YC, Xu L, Guo YL, Su HJ, Smith TJ, Ryan LM, Lee MS, Christiani DC. Polymorphisms in GSTT1 and p53 and urinary transitional cell carcinoma in south-western Taiwan: a preliminary study. Biomarkers. 2004;9:386-94.

22. Verma NHK, Manna SK. Advanced glycation end products (AGE) potentiates cell death in p53 negative cells via upregulation of NF-kappa B and impairment of autophagy. J Cell Physiol. 2017;232:3598-610.

23. He F, Mo L, Zheng XY, Hu C, Lepor H, Lee EYP, Sun TT, Wu XR. Deficiency of $\mathrm{pRb}$ family proteins and p53 in invasive urothelial tumorigenesis. Cancer Res. 2009;69:9413-21

24. Hoe KK, Verma CS, Lane DP. Drugging the p53 pathway: understanding the route to clinical efficacy. Nat Rev Drug Discov. 2014;13:217-36.

25. Silva JL, Gallo CVDM, Costa DCF, Rangel LP. Prion-like aggregation of mutant p53 in cancer. Trends Biochem Sci. 2014;39:260-7.

26. Wei $\mathrm{CH}, \mathrm{Wu}$ G, Cai Q, Gao XC, Tong F, Zhou R, Zhang RG, Dong JH, Hu Y, Dong XR. MicroRNA-330-3p promotes cell invasion and metastasis in non-small cell lung cancer through GRIA3 by activating MAPK/ERK signaling pathway. J Hematol Oncol. 2017;10:125.

27. Chatterjee SJ, Datar R, Youssefzadeh D, George B, Goebell PJ, Stein JP, Young L, Shi SR, Gee C, Groshen S, et al. Combined effects of p53, p21, and $\mathrm{pRb}$ expression in the progression of bladder transitional cell carcinoma. J Clin Oncol. 2004;22:1007-13.

28. Zhu Y, Dai B, Zhang H, Shi G, Shen Y, Ye D. Long non-coding RNA LOC572558 inhibits bladder cancer cell proliferation and tumor growth by regulating the AKT-MDM2-p53 signaling axis. Cancer Lett. 2016:380:369-74

29. Zhu HB, Yang K, Xie YQ, Lin YW, Mao QQ, Xie LP. Silencing of mutant p53 by siRNA induces cell cycle arrest and apoptosis in human bladder cancer cells. World J Surg Oncol. 2013;11:22. 